\title{
Parallel Semantic Trajectory Similarity Join
}

\author{
Lisi Chen ${ }^{b 1}$, Shuo Shang ${ }^{b 2}{ }^{*}$, Christian S. Jensen ${ }^{\ddagger 3}$, Bin Yao ${ }^{\sharp 4}$, Panos Kalnis ${ }^{\ddagger 5}$ \\ ${ }^{b}$ UESTC, China $\left\{{ }^{1}\right.$ chenlisi.cs; ${ }^{2}$ jedi.shang\}@gmail.com \\ $\ddagger$ Aalborg University, Denmark ${ }^{3}$ CS j@Cs.aau.dk \\ \# Shanghai Jiao Tong University, China ${ }^{4}$ yaobinecs. sjtu.edu.cn \\ ${ }^{\dagger}$ KAUST, Saudi Arabia ${ }^{5}$ panos. kalnis@kaust.edu.sa
}

\begin{abstract}
Matching similar pairs of trajectories, called trajectory similarity join, is a fundamental functionality in spatial data management. We consider the problem of semantic trajectory similarity join (STS-Join). Each semantic trajectory is a sequence of Points-of-interest (POIs) with both location and text information. Thus, given two sets of semantic trajectories and a threshold $\theta$, the STS-Join returns all pairs of semantic trajectories from the two sets with spatio-textual similarity no less than $\theta$. This join targets applications such as term-based trajectory near-duplicate detection, geo-text data cleaning, personalized ridesharing recommendation, keyword-aware route planning, and travel itinerary recommendation.
\end{abstract}

With these applications in mind, we provide a purposeful definition of spatio-textual similarity. To enable efficient STSJoin processing on large sets of semantic trajectories, we develop trajectory pair filtering techniques and consider the parallel processing capabilities of modern processors. Specifically, we present a two-phase parallel search algorithm. We first group semantic trajectories based on their text information. The algorithm's pergroup searches are independent of each other and thus can be performed in parallel. For each group, the trajectories are further partitioned based on the spatial domain. We generate spatial and textual summaries for each trajectory batch, based on which we develop batch filtering and trajectory-batch filtering techniques to prune unqualified trajectory pairs in a batch mode. Additionally, we propose an efficient divide-and-conquer algorithm to derive bounds of spatial similarity and textual similarity between two semantic trajectories, which enable us prune dissimilar trajectory pairs without the need of computing the exact value of spatio-textual similarity. Experimental study with large semantic trajectory data confirms that our algorithm of processing semantic trajectory join is capable of outperforming our well-designed baseline by a factor of 8-12.

\section{INTRODUCTION}

Trajectory similarity join is a fundamental functionality in trajectory data analytics (e.g., TS-Join [31], Strain-Join [38], DITA [36], DTJ [39]): Given sets $T$ and $Q$ of trajectories and a similarity threshold $\theta$, finds all pairs of trajectories from $T$ and $Q$ with a similarity that no less than $\theta$. Based on existing studies, the similarity between two trajectories is either measured by the spatial proximity [36], [38] or measured by spatio-temporal proximity [31], [39] between them, underlying that the join operation is conducted in spatial or spatio-temporal domain only.

However, recent years have witnessed the development of online map-based services (e.g., Bing Maps ${ }^{1}$, Google Maps ${ }^{2}$,

\footnotetext{
* Shuo Shang is the corresponding author.

${ }^{1}$ https://www.bing.com/maps/

${ }^{2}$ https://maps.google.com/
}

Foursquare $^{3}$, and MapQuest ${ }^{4}$ ) and location-based social media (e.g., Twitter ${ }^{5}$, Facebook $^{6}$, Instagram $^{7}$, and Flickr ${ }^{8}$ ). These applications are redefining and enriching the traditional trajectory data by associating locations with semantic meanings [48]. For example, Foursquare users can check in the Point-of-Interests (POI, which contains a spatial location and a semantic description) they are visiting and leave descriptions and comments for other users. Flickr allows tourists to upload their geo-text photos taken by smartphones during the travel, so that their trips can be outlined both by location and by semantic information embedded in the photos. Trajectories generated in these applications are defined as semanic trajectories [27]. They can be modeled either by a finite sequence of text-embedded geographical points (i.e., geo-textual objects, POIs) [17] or by a traditional trajectory enriched by a text document [34]. From a semantic trajectory, we can know not only where a user has been, as from the traditional trajectory, but also what he/she has done by retrieving the text documents associated with the locations (e.g., reviews, descriptions, text annotations), or by extracting the text information from the multimedia contents attached to the locations (e.g., images, videos).

To enable effective analytics of big semantic trajectory data, it is insufficient to evaluate the similarity between semantic trajectories solely based on spatial proximity. Consider semantic trajectories $\tau_{1}-\tau_{4}$ in Figure 1. The yellow diamonds represent the POIs covered by these trajectories. We see that trajectory $\tau_{1}$ covers a couple of tolled road segments, $\tau_{2}$ covers nearby free road segments with lake sightseeing, and $\tau_{3}$ covers some off-road segments. Although these three routes are spatially close to each other, they are preferred by diverging groups. In particular, $\tau_{1}$ is likely to be chosen by business travelers and commuters in a rush, $\tau_{2}$ is favorable to some budget-sensitive tourists, and $\tau_{3}$ may be taken by adventurous travelers who have off-road vehicles. Further, we see that $\tau_{4}$ is more similar to $\tau_{1}$ and $\tau_{3}$ than to $\tau_{2}$ in terms of spatial proximity. However, taking both trajectory spatial information and semantic (textual) information of POIs into account, we may find that travelers who choose $\tau_{2}$ are more likely to favor $\tau_{4}$ over $\tau_{1}$ and $\tau_{3}$. This example underlines that it is important to consider both spatial proximity and semantic similarity when determining whether two trajectories are similar with each other.

\footnotetext{
${ }^{3}$ https://foursquare.com

${ }^{4}$ https://www.mapquest.com

${ }^{5}$ https://twitter.com

${ }^{6}$ https://www.facebook.com

${ }^{7}$ https://instagram.com

${ }^{8}$ https://flickr.com
} 


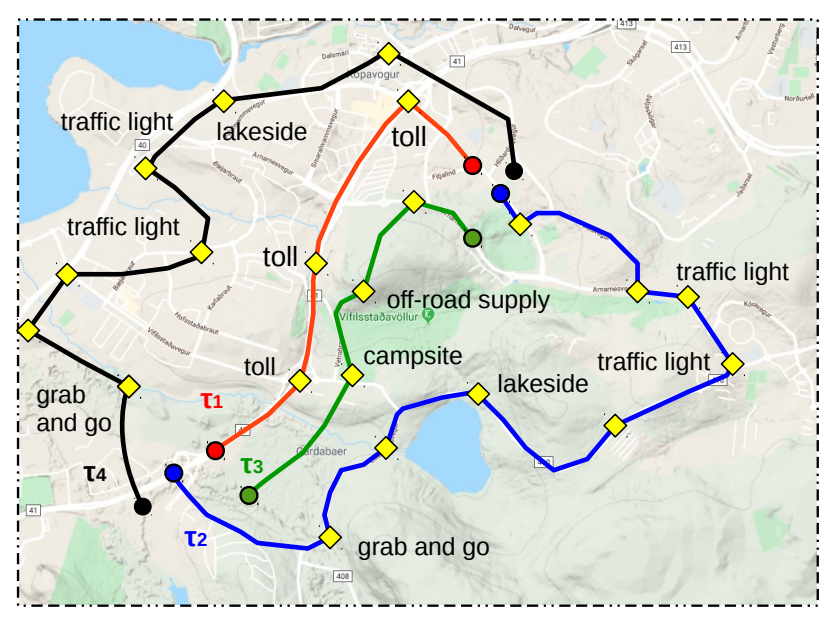

Fig. 1. An example of semantic trajectories

In this paper, we focus on a fundamental functionality in semantic trajectory data analytics, Semantic Trajectory Similarity Join (STS-Join): Given sets $T$ and $Q$ of semantic trajectories (or a single set $T$ and its mirror $Q$ in the case of self-join) and a similarity threshold $\theta$, the STS-Join returns all pairs of semantic trajectories from $T$ and $Q$ with a spatiotextual similarity that no less than $\theta$. Specifically, both spatial and textual domains are considered in measuring the similarity between two semantic trajectories. A linear combination method (e.g., [34], [47]) is adopted to combine the spatial and textual similarity into a spatio-textual similarity metric.

To process the STS-Join efficiently, we develop a twophase parallel matching framework that consists of the following techniques:

(1) Trajectory grouping and batch filtering: Given sets $T$ and $Q$ of trajectories, we first group trajectories by a purposeful inverted index based on their text information. The inverted index takes into account the parallel processing capabilities of modern processors. We prove that join processes of trajectories in different groups (lists) are independent of each other. Next, trajectories in each group are further partitioned according to the spatial domain. We generate spatial and textual summaries for each trajectory batch, based on which we propose batch filtering and trajectory-batch filtering techniques to prune unqualified trajectory pairs in a batch mode. (Sections IV-D)

(2) Semantic trajectory pair pruning: It is computationally expensive to calculate the exact spatio-textual similarity between two semantic trajectories (see Section III-C for complexity analysis). To address the challenge, we propose an efficient divide-and-conquer algorithm (see Section IV-C for detailed complexity analysis) to derive upper bounds of spatial similarity and textual similarity between two semantic trajectories, which enable us prune dissimilar trajectory pairs without the need of computing the exact value of spatio-textual similarity. (Sections IV-B and IV-C)

To sum up, the contributions of the paper are as follow.

- We present the first study on semantic trajectory similarity join, named STS-Join, that takes into account both spatial similarity and text similarity between trajectories.
- The STS-Join uses new metrics to evaluate semantic trajectory similarity in the spatial and textual domains.

- We develop a two-phase parallel matching framework consisting of semantic trajectory grouping, batch filtering, and trajectory pair pruning techniques that enable efficient parallel STS-Join processing.

- We conduct extensive experiments on large semantic trajectory data sets to study the performance of the algorithms.

The rest of the paper is organized as follows. Section II introduces two definitions of semantic trajectories and the similarity metrics used in the paper, and it defines the STSJoin problem. Section III presents the baseline algorithm for processing the STS-Join. Our two-phase parallel matching algorithm and corresponding filtering and pruning techniques are presented in Section IV. Next, we present our experimental study in Section V. Related work is covered in Section VI, and conclusions are presented in Section VII.

\section{Preliminaries AND Problem Statement}

\section{A. Semantic trajectories}

We present two popular definitions of semantic trajectories: POI-based semantic trajectory (e.g., [13], [17], [47], [48]) and document-based semantic trajectory (e.g., [34]).

Definition 1: (POI-based semantic trajectory) A POI-based semantic trajectory $\tau$ is a directed finite sequence of POIs $\left\langle o_{1}, o_{2}, \ldots, o_{n}\right\rangle$, where $o_{i}=\{\rho, \psi\}$ is a geo-textual object that contains a geographical location $\rho$ and a text description $\psi$.

Definition 2: (Document-based semantic trajectory) A document-based semantic trajectory $\tau=\{\mathbf{p}, \psi\}$ is defined by a directed finite location sequence $\mathbf{p}=\left\langle p_{1}, p_{2}, \ldots, p_{n}\right\rangle$ and a text document $\psi$.

For document-based semantic trajectory, the spatial information (location sequence) and text information are independent of each other. In contrast, the text information of POI-based semantic trajectory is associated with a particular POI, making the trajectory pair matching process be more challenging. As a result, in the remaining parts of this paper we focus on the similarity join of POI-based semantic trajectory. Our proposal can be easily extended to support documentbased semantic trajectory similarity join.

\section{B. Similarity measures}

In this section, we first present the definition of relevance between a geo-textual object and a semantic trajectory, based on which we present how to measure the similarity between two semantic trajectories.

1) Object-trajectory relevance: Given a geo-textual object $o$ and a semantic trajectory $\tau$, the spatio-textual relevance $\mathrm{R}(o, \tau)$ between $o$ and $\tau$ are defined as follow. ${ }^{9}$

$$
\mathrm{R}(o, \tau)=\max _{o_{i} \in \tau}\left\{\operatorname{Sim}\left(o, o_{i}\right)\right\}
$$

\footnotetext{
9 Unless otherwise stated, semantic trajectory denotes POI-based semantic trajectory.
} 
where $\operatorname{Sim}\left(o, o_{i}\right)$ denotes the spatio-textual similarity between $o$ and $o_{i}$, which is computed by a linear combination of spatial proximity and text similarity [5], [18], [43], [44]:

$$
\operatorname{Sim}\left(o_{1}, o_{2}\right)=\alpha \cdot \mathrm{S}\left(o_{1} \cdot \rho, o_{2} \cdot \rho\right)+(1-\alpha) \cdot \mathrm{T}\left(o_{1} \cdot \psi, o_{2} \cdot \psi\right)
$$

where $\mathrm{S}\left(o_{1} . \rho, o_{2} . \rho\right)$ denotes the normalized spatial score between $o_{1} . \rho$ and $o_{2} . \rho$, which is inversely proportional to the distance, and $\mathrm{T}\left(o_{1} . \psi, o_{2} . \psi\right)$ denotes the normalized text similarity (e.g., cosine similarity, Jaccard similarity) between $o_{1} . \psi$ and $\mathrm{O}_{2} . \psi$. Without loss of generality, we adopt Jaccard similarity (cf. Equation 3), which is a widely-used model to measure the similarity between geo-textual objects [10], [15], [18], [22]. It is important to note that our proposal is not specific to the Jaccard similarity measurement. It is straightforward to extend our method to other similarity measure such as cosine similarity [40], [44]. Parameter $\alpha$ balances the weight between spatial proximity and text similarity.

$$
\mathrm{T}\left(o_{1} . \psi, o_{2} . \psi\right)=\frac{\left|o_{1} . \psi \cap o_{2} . \psi\right|}{\left|o_{1} . \psi \cup o_{2} . \psi\right|}
$$

2) Trajectory-trajectory similarity: Given semantic trajectories $\tau_{1}$ and $\tau_{2}$, we define the spatio-textual similarity $\operatorname{ST}\left(\tau_{1}, \tau_{2}\right)$ between them based on an existing study [12].

$$
\operatorname{ST}\left(\tau_{1}, \tau_{2}\right)=\frac{\sum_{o_{i} \in \tau_{1}} \mathrm{R}\left(o_{i}, \tau_{2}\right)}{\left|\tau_{1}\right|}+\frac{\sum_{o_{j} \in \tau_{2}} \mathrm{R}\left(o_{j}, \tau_{1}\right)}{\left|\tau_{2}\right|}
$$

Here, $\tau$ denotes the number of geo-textual objects in a semantic trajectory. We ensure that the similarity measures are symmetrical, such that $\operatorname{ST}\left(\tau_{1}, \tau_{2}\right)=\operatorname{ST}\left(\tau_{2}, \tau_{1}\right)$.

\section{Problem Definition}

We formally define the STS-Join problem in Definition 3.

Definition 3: (STS-Join) Given sets $T$ and $Q$ of semantic trajectories and a similarity threshold $\theta$, the semantic trajectory similarity join (STS-Join) finds a set $A$ of all semantic trajectory pairs $\left(\tau_{i}, \tau_{j}\right)$ from the two sets that satisfy the following conditions:

(1) $\tau_{i}$ and $\tau_{j}$ share at least one common term, and

(2) The spatio-textual similarity between $\tau_{i}$ and $\tau_{j}$ is no less than $\theta$.

Condition (1) in Definition 3 is to guarantee that the trajectory pairs in each join result have some minimum textual relevancy to each other. Specifically, if two semantic trajectories $\tau_{1}$ and $\tau_{2}$ are to be considered as similar, a very natural necessary condition is that $\tau_{1}$ and $\tau_{2}$ have some minimum overlap in both the spatial and textual domains. This setting has been applied widely in existing studies that measure the similarity between two geo-textual items (e.g., [3], [16], [21]).

Complexity analysis. We assume that the number of objects in each trajectory is $\left|\tau_{a v g}\right|$ and that the number of terms per object is $\left|\psi_{\text {avg }}\right|$. According to Equations 1 and 4, to compute $\mathrm{ST}\left(\tau, \tau_{j}\right)$ we need to calculate the spatio-textual similarity between each pair of objects in $\tau$ and $\tau_{j}$. So the time complexity of computing $\mathrm{ST}\left(\tau, \tau_{j}\right)$ is $O\left(\left|\psi_{\text {avg }}\right| \cdot\left|\tau_{\text {avg }}\right|^{2}\right)$. In the worst case, we need to evaluate all pairs of trajectories. Thus, the time complexity of the STS-Join is $O\left(\left|\psi_{\text {avg }}\right| \cdot\left|\tau_{\text {avg }}\right|^{2} \cdot|T|^{2}\right)$.

We initially consider the self-join scenario (i.e., $T=Q$ ), and then cover the case $T \neq Q$ in Section IV-E.

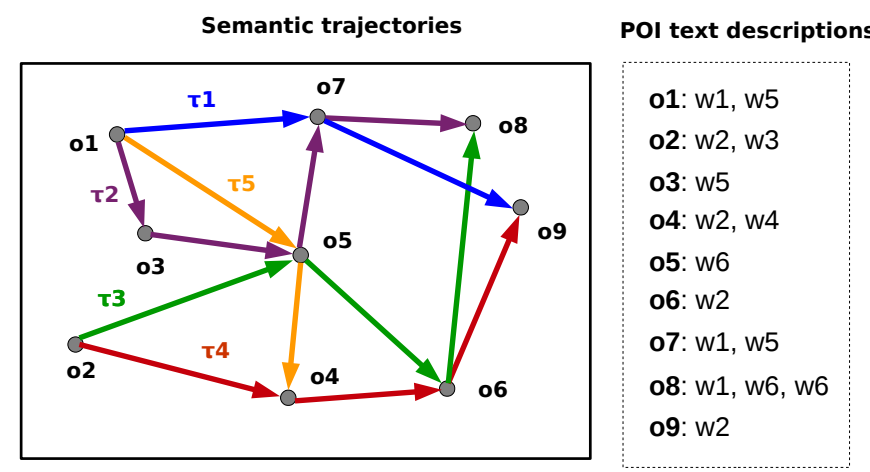

Fig. 2. An example of semantic trajectory collection

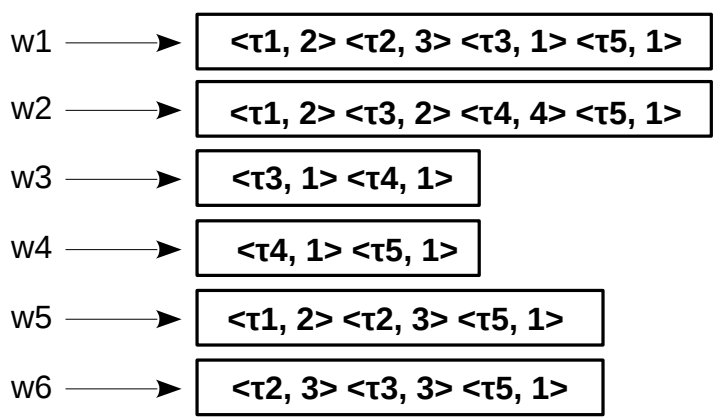

Fig. 3. Trajectory inverted file

\section{BASELINE APPROACH}

\section{A. Basic idea}

Search with Trajectory Inverted File (STIF) is a straightforward baseline approach to computing the STS-Join. Based on Definition 3, trajectory pair $\tau_{i}$ and $\tau_{j}$ can be a join result only if they share at least one common term. In order to filter out trajectory pairs that do not share any term, we index the trajectory terms using inverted index. In particular, each inverted list, denoted by $I L(w)$, corresponds to a term $w$ and it stores trajectories whose text descriptions contain $w$. Then we evaluate each trajectory pair that shares at least one common term. Specifically, for each trajectory $\tau$ we evaluate potential result pairs $\left(\tau, \tau^{\prime}\right)$ by traversing all inverted lists $I L\left(w_{i}\right)$ where $w_{i} \in W$ and $W=\cup_{o_{j} \in \tau} o_{j} . \psi$. We compute $\operatorname{ST}\left(\tau, \tau^{\prime}\right)$ and compare it with $\theta$.

\section{B. Trajectory Inverted File}

Figure 2 presents a collection of semantic trajectories. Gray circles $O_{1}-O_{9}$ are POIs and $\tau_{1}-\tau_{5}$ are semantic trajectories. Each trajectory is illustrated by a unique color. The text description of each POI is presented on right-hand side.Figure 3 presents the trajectory inverted file built for indexing the trajectories in Figure 2. Each posting $\left\langle\tau_{i}, n\right\rangle$ in $I L(w)$ consists of a trajectory id, $\tau_{i}$, and the frequency of $w$ in $\tau_{i}, n$. Note that the frequency may be used for computing the spatiotextual similarity between two trajectories, depending on the text similarity metric we use. 


\section{Algorithm}

This section presents our STIF algorithm (Algorithm 1). The inputs are a collection of trajectories $T$ and a similarity threshold $\theta$. The output is a set of trajectory pairs that are similar with each other. For each trajectory $\tau$, we first generate a union of terms in all of its POIs (i.e., $W$ ) (Line 2). Next, for each term $w_{i} \in W$ we traverse its inverted list $I L\left(w_{i}\right)$ and evaluate each posting $\left\langle\tau_{j}, n\right\rangle$ in $I L\left(w_{i}\right)$ (Line 3). In particular, we compute the similarity between $\tau$ and $\tau_{j}$. If the similarity is no less than $\theta$, we add pair $\left(\tau, \tau_{j}\right)$ to the result set $A$ (Lines 4-7). At the end, we return set $A$ as the final result. In this algorithm, the evaluation of each trajectory $\tau$ can be performed in parallel.

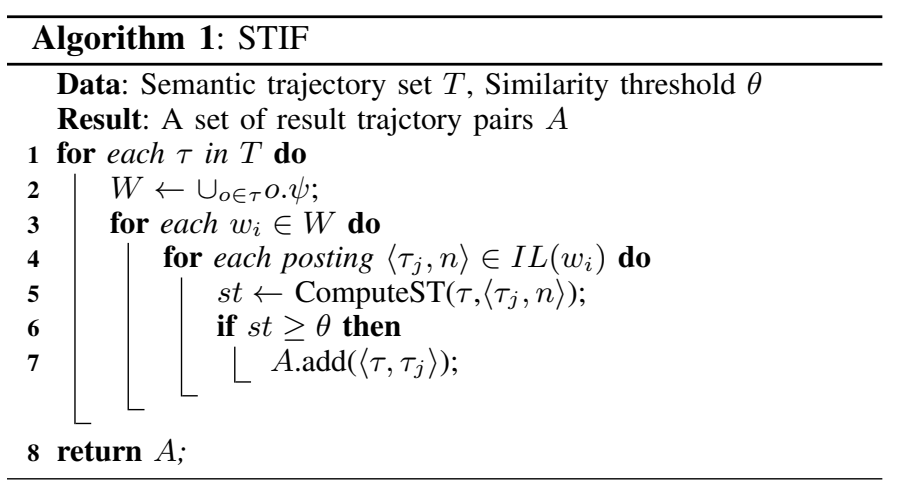

\section{Two-Phase Parallel Matching}

\section{A. Basic Idea}

We aim to address the following limitations exist in STIF algorithm:

(1) High complexity of computing similarity between two trajectories: When computing ST $\left(\tau_{i}, \tau_{j}\right)$ we need to calculate the similarity between object pair $\left\langle o_{i}, o_{j}\right\rangle$ s.t. $o_{i} \in \tau_{i}$ and $o_{j} \in \tau_{j}$. It is computationally expensive especially when the average number of objects in a trajectory is large.

(2) Individual evaluation: In STIF, each trajectory pair is evaluated separately, which is time consuming given a large number of semantic trajectories.

To address the above two limitations, we develop a Twophase Parallel Matching (2-PM) algorithm for processing STS-Join. Specifically, to address limitation (1) we propose an trajectory pair pruning strategy to prune "unqualified" trajectory pairs without the need of calculating the similarity between each object pair in $\tau_{i}$ and $\tau_{j}$. To address limitation (2), we propose a parallelized batch processing algorithm that is able to evaluate a group of trajectory pairs simultaneously.

Figure 4 presents the framework of our 2-PM algorithm. Given a collection of semantic trajectories $T$, we generate summary for each trajectory to represent its spatial and textual information (cf. Section IV-B). Based on the summary information we group similar trajectories together and conduct batch filtering (cf. Section IV-D). Specifically, if a pair of batches (groups) cannot be pruned, we proceed to evaluate each trajectory pair in the two batches by using our trajectory pair filtering technique (cf. Section IV-C). Note that both batch filtering and trajectory pair filtering can be processed in parallel.

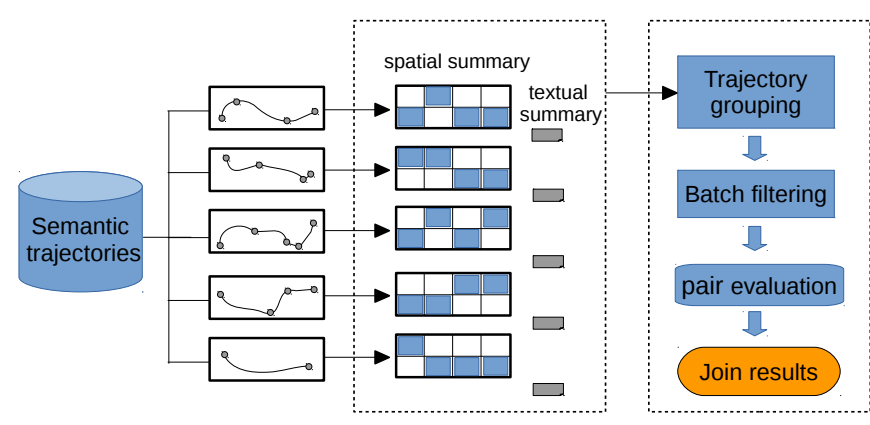

Fig. 4. Framework of 2-PM

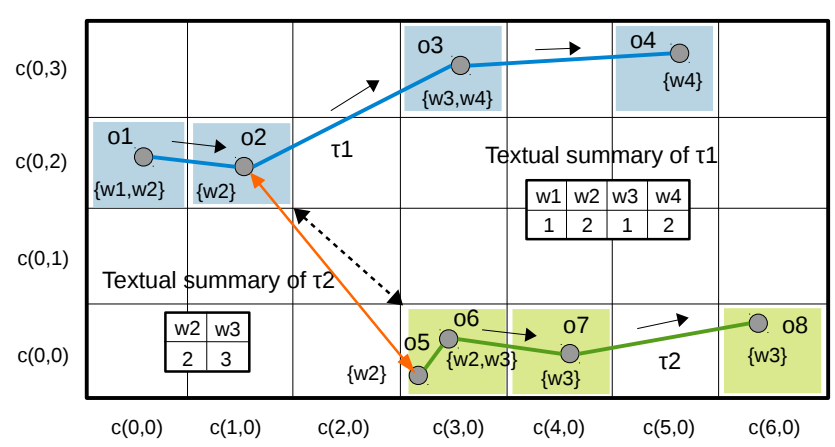

Fig. 5. Summary of trajectories

\section{B. Semantic Trajectory Summarization}

This section presents how to generate a summarization of a semantic trajectory. Recall that the time complexity of computing the spatio-textual similarity between two trajectories is $O\left(\left|\psi_{\text {avg }}\right| \cdot\left|\tau_{\text {avg }}\right|^{2}\right)$. With the summarizations of trajectories $\tau_{i}$ and $\tau_{j}$, we can derive an upper bound of spatio-textual similarity between $\tau_{i}$ amd $\tau_{j}$ with $O\left(\left|\tau_{\text {avg }}\right| \cdot \log \left|\tau_{\text {avg }}\right|+\left|\tau_{\text {avg }}\right|\right.$. $\left.\left|\psi_{\text {avg }}\right|\right) .^{10}$

Figure 5 presents an example of summarizing trajectories $\tau_{1}=\left\langle o_{1}, o_{2}, o_{3}, o_{4}\right\rangle$ and $\tau_{2}=\left\langle o_{5}, o_{6}, o_{7}, o_{8}\right\rangle$. We summarize the spatial information and textual information of trajectories separately. For summarizing spatial information, we partition the underlying space into $m \times n$ (i.e., $7 \times 4$ in Figure 5) cells. Each object is associated with a cell $c(x, y)$. As a result, trajectory $\tau_{1}$ is associated with $c(0,2), c(1,2), c(3,3)$, and $c(5,3)$, and trajectory $\tau_{2}$ is associated with $c(3,0), c(4,0)$, and $c(6,0)$. As for summarizing textual information, we aggregate the term frequencies of all objects in each trajectory (see textual summaries of $\tau_{1}$ and $\tau_{2}$ in Figure 5).

\section{Trajectory Pair Pruning}

We proceed to introduce how to derive upper bounds of spatial similarity and textual similarity between two trajectories, respectively, which enable us prune "dissimilar" trajectory pairs without the need of computing the exact value of spatiotextual similarity.

1) Spatial similarity upper bound: Computing the spatial similarity upper bound between trajectories $\tau_{i}$ and $\tau_{j}$, denoted by $\mathrm{ST}_{S}\left(\tau_{i}, \tau_{j}\right) . u b$, is equivalent to computing the lower bound

\footnotetext{
${ }^{10}$ Assume that the trajectory is not summarized by grid cells.
} 


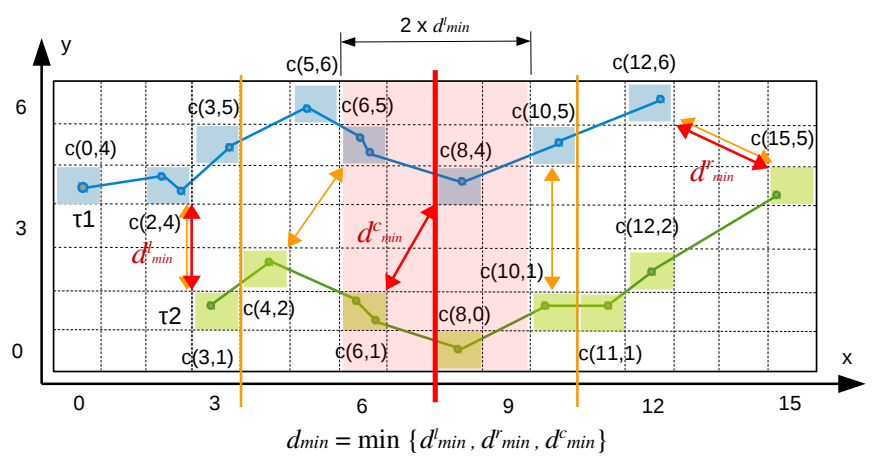

Fig. 6. Computation of spatial similarity upper bound

of minimum distance between $\tau_{i}$ and $\tau_{j}$, which is denoted by $d_{\text {min }}$.

Figure 6 illustrates the high-level idea of our divide-andconquer based algorithm of computing $d_{\text {min }}$. We first index $\tau_{1}$ and $\tau_{2}$ by grid cells. Next, we recursively calculate the local minimum distances between cells of $\tau_{1}$ and $\tau_{2}$ in both subareas divided by the vertical line (i.e., both left and right subareas contain the same number of cells). Specifically, in the first recursion, the red boundary line parallel to y-axis in Figure 6 partitions the underlying space into two subspaces. $d_{\text {min }}^{l}$ and $d_{m i n}^{r}$, represented by double-headed red arrow lines, denotes the local minimum distances regarding the left subspace and right subspace, respectively. After calculating the local minimum distances regarding both subspaces, we compute $d_{m i n}^{c}$, the local minimum distance between cells of $\tau_{1}$ and $\tau_{2}$ in the area located at $\operatorname{most} \min \left\{d_{\text {min }}^{l}, d_{\min }^{r}\right\}$ distance away from the middle line dividing the two subspaces (i.e., the red area in Figure 6).

Algorithm 2 presents our divide-and-conquer algorithm of computing the lower bound of minimum distance given trajectories $\tau_{1}$ and $\tau_{2}$. At first, we index $\tau_{1}$ and $\tau_{2}$ by grid cells (Lines 1-2). In particular, $C_{1}$ and $C_{2}$ to denote the sets of grid cells associated with $\tau_{1}$ and $\tau_{2}$, respectively, and array $A$ (Line 3) is a list of cells that merges $C_{1}$ and $C_{2}$. Next, we sort $A$ based on x-coordinate and y-coordinate and generate arrays $A_{x}$ and $A_{y}$, respectively. Finally, we call recursive function MinDistUtil by taking $C_{1}, C_{2}, A_{x}, A_{y}$, and $|A|$ as input.

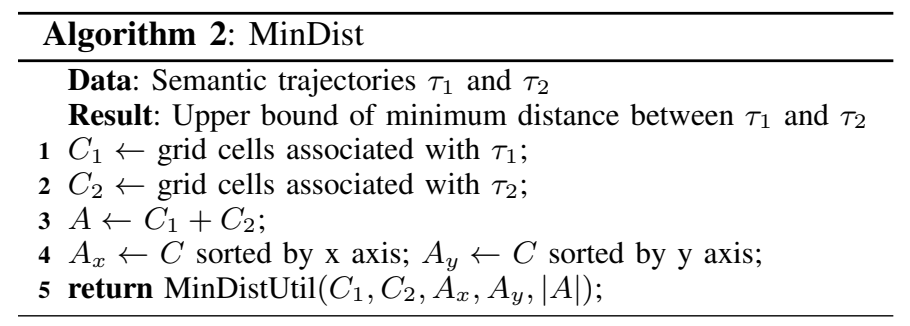

We proceed to present the pseudo code of MinDistUtil in Algorithm 3. Here, integer $m$ represents the total number of cells associated with $\tau_{1}$ and $\tau_{2}$ (i.e., $\left|C_{1}\right|+\left|C_{2}\right|$ ). At first, we check if $m$ is large enough to use divide-and-conquer framework. If $m \leq 3$, we directly compute $d_{\min }$ in a brute force manner by enumerating all pairs of cells (Lines 31-32). If $m>3$, we go with our divide-and-conquer method. Let $A_{x}^{l}$ and $A_{x}^{r}$ be the first and second half of $A_{x}$, respectively
(Lines 6-7). We split $A_{y}$ into $A_{y}^{l}$ and $A_{y}^{r}$ according to xcoordinate (Lines 8-12). Next, we recursively calculate the local $d_{\min }$ regarding $A_{y}^{l}$ and $A_{y}^{r}$. In particular, if $A_{y}^{l}$ (resp. $A_{y}^{r}$ ) contains cells associated with both $\tau_{1}$ and $\tau_{2}$, we compute the local minimum distance $d_{\min }^{l}$ (resp. $d_{\min }^{r}$ ) by calling the next recursion of MinDistUtil; otherwise, we assign the initial value $(\max )$ to $d_{\min }^{l}\left(\right.$ resp. $\left.d_{\min }^{r}\right)$ as the local minimum distance does not exist (Lines 13-20). Having $d_{\min }^{l}$ and $d_{\min }^{r}$, we choose the smaller one as the minimum distance regarding the left subspace and right subspace (Line 21). Following that, we compute the local minimum distance between cells close to the boundary line in the current recursion (Lines 2230). Specifically, we generate array $A_{m}$, sorted based on ycoordinate, that contains cells whose distances to the boundary line are closer than $d_{\min }$ (Lines 23-25). Then we evaluate all pairs of cells from different trajectories and update the value of $d_{\min }$ (Lines 26-30). It is worth noting that the complexity of cross-boundary computation (i.e., Lines 26-30) is not $O\left(\left(\left|C_{1}\right|+\left|C_{2}\right|\right)^{2}\right)$. Instead, it is $O\left(\left(\left|C_{1}\right|+\left|C_{2}\right|\right)\right)$ because the inner loop runs at most 7 times. We regard each cell as a point in order to prove the above proposition in a more understandable way (cf. Theorem 1).

Theorem 1: The number of inner loops (Lines 27-30 in Algorithm 3) in the cross-boundary computation is at most 7.

Proof: Given a point $p_{0}$ that is visited by a particular outer loop (Lines 26-30 in Algorithm 3). The points visited by the corresponding inner loop (Lines 27-30) must lie either in the left or in the right $d_{\min } \times d_{\min }$ square. Because that for any two points $p_{i}$ and $p_{j}$, the distance between $p_{i}$ and $p_{j}$ is larger than $d_{m i n}$, we can pack at most 4 points into one square. Thus, we have 7 points in total expect $p_{0}$.

Complexity Analysis. Given two sets of cells $C_{1}$ and $C_{2}$ associated with trajectories $\tau_{1}$ and $\tau_{2}$, respectively, the time complexity of MinDist algorithm is $O\left(\left(\left|C_{1}\right|+\left|C_{2}\right|\right) \cdot \log \left(\left|C_{1}\right|+\right.\right.$ $\left.\left.\left|C_{2}\right|\right)\right)$.

2) Textual similarity upper bound: We proceed to compute the upper bound of text similarity between trajectories $\tau_{1}$ and $\tau_{2}$ based on Equation 5 .

$\mathrm{ST}_{T}\left(\tau_{1}, \tau_{2}\right) \cdot u b=\frac{\sum_{o_{i} \in \tau_{1}} \mathbf{R}_{T}\left(o_{i}, \tau_{2}\right) \cdot u b}{\left|\tau_{1}\right|}+\frac{\sum_{o_{j} \in \tau_{2}} \mathbf{R}_{T}\left(o_{j}, \tau_{1}\right) \cdot u b}{\left|\tau_{2}\right|}$,

where $\mathbf{R}_{T}\left(o_{i}, \tau\right) . u b$ denotes the upper bound of text relevance between object $o_{i}$ and trajectory $\tau$. Equation 5 calculates $\mathbf{S T}_{T}\left(\tau_{1}, \tau_{2}\right) . u b$ by aggregating the upper bound of text relevances between each $o_{i} \in \tau_{1}$ and $\tau_{2}$, and between each $o_{i} \in \tau_{2}$ and $\tau_{1}$.

Algorithm 4 presents the pseudo code of computing the upper bound of text similarity between $\tau_{1}$ and $\tau_{2}$. We use $t s(\tau)$ to denote the set of terms in trajectory $\tau$, which can be directly retrieved from the textual summary of $\tau$ (cf. Figure 5). We take the text summaries of $\tau_{1}$ and $\tau_{2}$ as input. At first, we initialize TSim, which maintains the current text similarity (Line 1). Then we aggregate TSim with the upper bound of text relevances between each $o_{i} \in \tau_{1}$ and $\tau_{2}$ (i.e., $\mathrm{R}_{T}\left(o_{i}, \tau_{2}\right) . u b$ ) (Lines 2-4). Next, we do the same with the upper bound of text relevances between each $o_{i} \in \tau_{2}$ and $\tau_{1}$ (i.e., $\mathbf{R}_{T}\left(o_{i}, \tau_{1}\right) . u b$ ) (Lines $5-7$ ). We can see that the time 

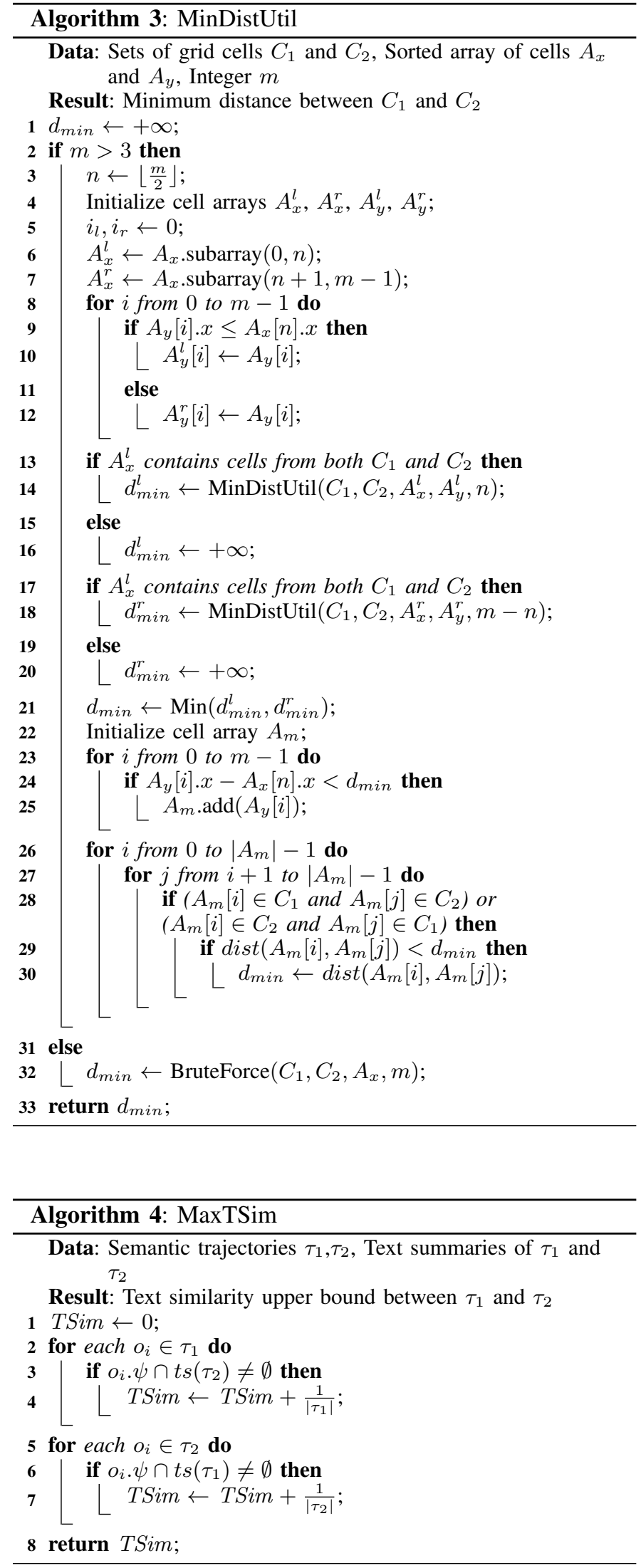

complexity of computing $\mathrm{R}_{T}\left(o_{i}, \tau_{1}\right) . u b$ and $\mathrm{R}_{T}\left(o_{i}, \tau_{2}\right) . u b$ is

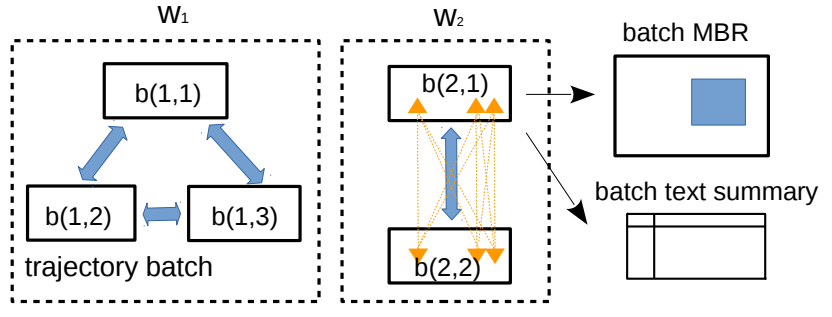

Fig. 7. Trajectory grouping

$O\left(\left|o_{i} . \psi\right|\right)$ and $O\left(\left|o_{i} \cdot \psi\right|\right)$, respectively. ${ }^{11}$

\section{Trajectory Grouping and Batch Filtering}

In our semantic trajectory join setting, the number of semantic trajectories can be very large, making it very time consuming to evaluate each trajectory pair individually. So we propose to evaluate groups of spatially and textually similar trajectories simultaneously.

Figure 7 presents our framework of trajectory grouping scheme. Similar to the baseline approach introduced in Section III, semantic trajectories are firstly indexed by an inverted file. Each inverted list maintains trajectories that contain a particular term. For example, in Figure 7, trajectories maintained under $w_{i}$ must contain term $w_{i}$ in at least one of their object. Trajectories in each inverted list are grouped into batches based on their spatial information (see Section IV-D4 for grouping algorithm). Here, we use $b(i, j)$ to denote the $j$-th batch of trajectories in inverted list of term $w_{i}$ (i.e., $I L\left(w_{i}\right)$ ). Each batch is augmented with batch MBR and batch text summary. In particular, the batch MBR is a minimum bounding rectangle that enclose the objects of all trajectories in the batch and the batch text summary summarize the terms of trajectories in the batch.

Based on our trajectory grouping scheme, we develop the join algorithm consisting of the following three phases.

(1) Intra-list batch filtering: Given $I L\left(w_{i}\right)$, we first evaluate each pair of batches in $I L\left(w_{i}\right)$ (e.g., $b(i, j)$ and $b(i, k)$ ) and check if there may exist $\tau \in b(i, j)$ and $\tau^{\prime} \in b(i, k)$ s.t. $\mathrm{ST}\left(\tau, \tau^{\prime}\right) \geq \theta$. If not, batch pair $b(i, j)$ and $b(i, k)$ can be filtered out. Note that it is unnecessary to evaluate batch pair $b(x, j)$ and $b(y, k)$ if $x \neq y$ (cf. Theorem 2). The upper bound of similarity between two batches is introduced in Section IV-D1

(2) Trajectory-batch filtering: If batch pair $b(i, j)$ and $b(i, k)$ cannot be filtered, we proceed to evaluate each trajectory $\tau \in b(i, j)$. Specifically, we check if there may exist $\tau^{\prime} \in b(i, k)$ s.t. ST $\left(\tau, \tau^{\prime}\right) \geq \theta$. If not, trajectory-batch pair $\tau$ and $b(i, k)$ can be filtered out. The upper bound of similarity between a trajectory and a batch is introduced in Section IV-D2

(3) Individual evaluation: If trajectory-batch pair $\tau$ and $b(i, k)$ cannot be filtered, we need to evaluate each trajectory pair of $\tau$ and $\forall \tau^{\prime} \in b(i, k)$. Next, we evaluate each pair in $b(i, j)$. Trajectory pair pruning technique (cf. Section IV-C) is applied in individual evaluation.

\footnotetext{
${ }^{11}$ The complexity result is based on the fact that the textual summary of each trajectory is maintained by hashmap.
} 
Theorem 2: Given $b(x, j)$ and $b(y, k)$, batch pair $b(x, j)$ and $b(y, k)$ can be filtered out if $x \neq y$.

Proof: Let $\tau_{a} \in b(x, j)$ and $\tau_{b} \in b(y, k)$. We consider the following two scenarios: (1) There exist $o_{m} \in \tau_{a}$ and $o_{n} \in \tau_{b}$ s.t. $o_{m}$ and $o_{n}$ share common term $w_{q}$; (2) For any $o_{m} \in \tau_{a}$ and $o_{n} \in \tau_{b}, o_{m}$ and $o_{n}$ do not share common term. For scenario (1), we can deduce that both $\tau_{a}$ and $\tau_{b}$ are indexed by $I L\left(w_{q}\right)$. Thus, they must be evaluated in intra-list batch filtering phase. As for scenario (2), trajectory pair $\tau_{a}$ and $\tau_{b}$ can be safely pruned based on Definition 3 .

1) Spatio-textual similarity upper bound between two batches: Given batches $b_{i}$ and $b_{j}$ where $b_{i}, b_{j} \in I L(w)$, the spatio-textual similarity upper bound of trajectory pair $\left(\tau_{a}, \tau_{b}\right)$, where $\tau_{a} \in b_{i}$ and $\tau_{b} \in b_{j}$, is computed by Equation 6 .

$$
\mathrm{ST}_{B}\left(b_{i}, b_{j}\right) \cdot u b=\alpha \cdot \mathbf{S}_{\max }\left(b_{i} \cdot m b r, b_{j} \cdot m b r\right) \cdot 2+(1-\alpha) \cdot 2,
$$

where $\mathrm{S}_{\max }\left(b_{i} \cdot m b r, b_{j} . m b r\right)$ denotes the maximum spatial similarity between the minimum bounding rectangle (MBR) of trajectories in $b_{i}$ and the MBR of trajectories in $b_{j}$. If $\mathrm{ST}_{B}\left(b_{i}, b_{j}\right) \cdot u b<\theta$, we filter out all trajectory pairs in $b_{i}$ and $b_{j}$.

2) Spatio-textual similarity upper bound between a trajectory and a batch: Given trajectory $\tau$ and batch $b$, the spatiotextual similarity upper bound between $\tau$ and $b$ is computed by Equation 7 .

$$
\begin{aligned}
& \mathrm{ST}_{T B}(\tau, b) \cdot u b= \\
& \frac{\sum_{o_{i} \in \tau} \mathbf{R}_{\max }\left(o_{i}, b\right)}{|\tau|}+\alpha \cdot \mathbf{S}_{\max }(\tau, b . m b r)+1-\alpha
\end{aligned}
$$

$\mathrm{S}_{\max }\left(\tau, b_{j} . m b r\right)$ denotes the maximum spatial score between $\tau$ and the MBR of $b, \mathbf{R}_{\max }\left(o_{i}, b\right)$ denotes the maximum relevance between $o_{i}$ and trajectories in $b$, which is computed by Equation 8 .

$\mathrm{R}_{\max }\left(o_{i}, b\right)=\alpha \cdot \mathbf{S}_{\max }\left(o_{i} \cdot \rho, b \cdot m b r\right)+(1-\alpha) \cdot \mathbf{T}_{\max }\left(o_{i} \cdot \psi, b\right)$,

where $\mathrm{T}_{\max }\left(o_{i} . \psi, b\right)$ can be easily acquired by traversing the text summary of $b$. If $\mathrm{ST}_{T B}(\tau, b) . u b<\theta$, we filter out all pairs between $\tau$ and trajectories in $b$.

3) BFJoin Algorithm: Our join algorithm, BFJoin, can be performed in parallel. Based on Theorem 2, we only need to run trajectory join algorithm within each inverted list. Consequently, the join algorithms regarding different inverted lists are independent of each other.

Algorithm 5 presents the pseudo code of out join algorithm regarding inverted list $I L\left(w_{x}\right)$. The inputs are $I L\left(w_{x}\right)$ (including its MBR and text summary) and the similarity threshold $\theta$. The output is a set of similarity trajectory pairs within $I L\left(w_{x}\right)$, which is denoted by Result. We start our intra-list batch filtering phase (Lines 4-16). Specifically, we compute the spatio-textual similarity upper bound between batches $b(x, i)$ and $b(y, i)$ based on Equation 6 . If the upper bound is lower than $\theta$, we filter out batch pair $b(x, i)$ and $b(y, i)$. If the pair cannot be filtered, we proceed with the trajectory-batch filtering phase (Lines 6-16). To improve the pruning power, we compute the spatio-textual similarity upper bound between the batch that has smaller MBR (i.e., $b_{2}$ ) and each $\tau$ in the batch that has larger MBR (i.e., $b_{1}$ ) based on Equation 7. If the upper bound is not lower than $\theta$, we proceed

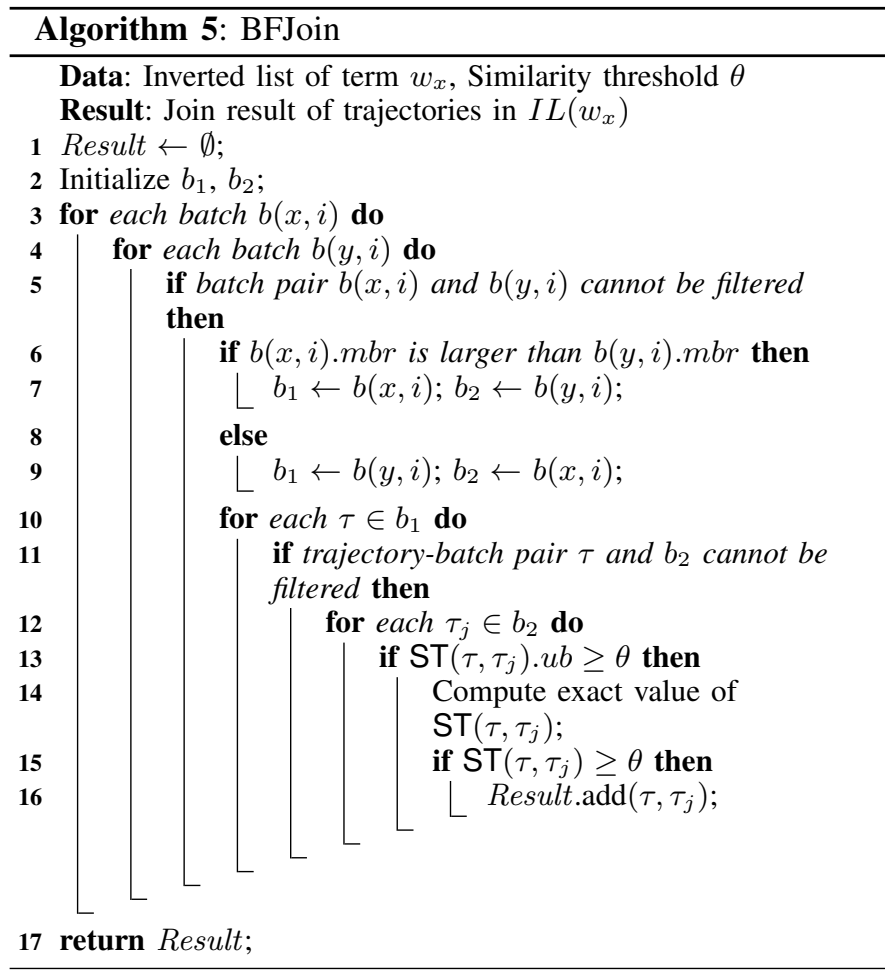

with our individual evaluation phase, which evaluates each pair of trajectories in $b_{1}$ and $b_{2}$ (Lines 12-16). In particular, we compute the similarity upper bound of a trajectory pair by using the trajectory pruning technique (cf. Section IV-C). If the upper bound is not lower than $\theta$, we need to calculate the exact value of the trajectory pair similarity (Line 14). If the similarity is not lower than $\theta$, we add the pair to Result (Lines 15-16).

4) Trajectory grouping algorithm: To improve the effectiveness of intra-list batch filtering and trajectory-batch filtering, we need to compute a more accurate value of maximum spatial score. Note that the maximum spatial score is equivalent to minimum distance. For this purpose, it is useful to maintain the "quality" of each batch $b$ in order to reduce the discrepancy between the minimum distance from object $o$ to $b . m b r$ (denoted by minDist $(o . \rho, b . m b r)$ ) and the exact distance from $o$ to $o^{\prime}$ (denoted by $\operatorname{dist}\left(o . \rho, o^{\prime} . \rho\right)$ ) where $o^{\prime} . r h o \in b . m b r$. According to triangle inequity theorem, it is easy to deduce that the maximum possible discrepancy between minDist $(o . \rho, b . m b r)$ and $\operatorname{dist}\left(o . \rho, o^{\prime} . \rho\right)$ is the diagonal length of $b . m b r$, which is denoted by $\mathrm{dg}(b . \mathrm{mbr})$.

To increase the accuracy of minDist $(o . \rho, b . m b r)$, we constrain the value of $\mathrm{dg}($ b.mbr $)$ for each batch. We develop a Diagonal-Constrained Online Grouping (DCOG) algorithm for grouping trajectories in an inverted list such that the MBR of trajectories in each batch $b$ satisfies $\operatorname{dg}(b . m b r) \leq \theta_{\text {diag }}$, where $\theta_{\text {diag }}$ is the threshold of MBR diagonal length.

Algorithm 6 presents the pseudo code of the DCOG algorithm. It takes the original inverted list $I L(w)$ and the threshold of MBR diagonal length $\theta_{\text {diag }}$ as input. For each trajectory $\tau \in I L(w)$, we perform online grouping. Specifically, we assign $\tau$ to the batch with MBR that has the shortest diagonal length. At first, we initialize $b_{\min }$ and $\operatorname{diag}_{\min }$, which 


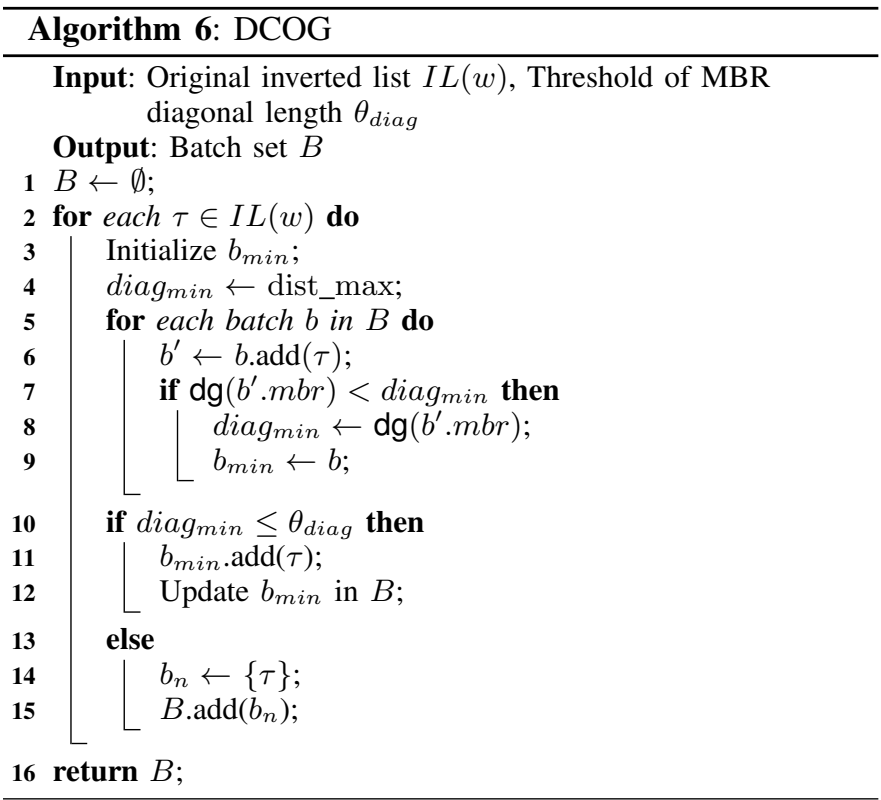

denote the current batch with the shortest diagonal length and the corresponding diagonal length, respectively (Lines 34). Next, for each batch $b$ in batch set $B$ we add $\tau$ to $b$, which is denoted by $b^{\prime}$ (Line 6). We find the batch $b_{\min }$ that has the minimum MBR diagonal length (Lines 7-9). If the corresponding diagonal length does not exceed the threshold $\theta_{\text {diag }}$, we assign $\tau$ to $b_{\min }$ and update $B$ (Lines 10-112; otherwise, we create a new batch $b_{n}$ with the single trajectory $\tau$ and add $b_{n}$ to $B$ (Lines 13-15). Finally, we return $B$ as the grouping result (Line 16).

\section{E. Extension of Non-self Join}

We proceed to explain how to extend our proposal to support non-self join, where we have two semantic trajectory sets $T$ and $Q(T \neq Q)$. We can extend the BFJoin algorithm to support the case where $T \neq Q$ in a straightforward manner by maintaining inverted file for trajectories in $T$ and $Q$ separately.

Let $I L_{T}(w)$ and $I L_{Q}(w)$ be inverted lists of term $w$ for $T$ and $Q$, respectively. We present our extension of BFJoin algorithm that supports non-self join (Algorithm 7).

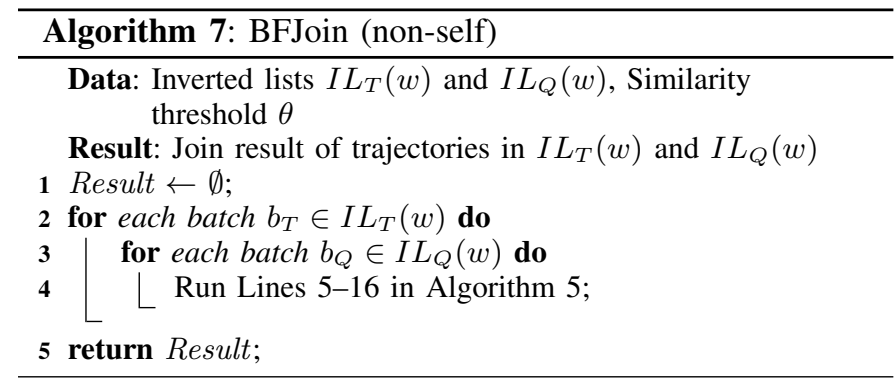

\section{EXPERIMENTAL STUDY}

\section{A. Experiment Settings}

1) Datasets: We use two datasets: New York trajectory data (NTD) ${ }^{12}$ and Beijing trajectory data (BTD). The NTD consists of a road network and 10M taxi trips. Each taxi trip is a source-destination pair. We regard the shortest path from the source to the destination as the trajectory of a trip. In addition, we use a real POI data set that contains 19,969 POIs in New York City ${ }^{13}$. Each POI has a spatial coordinate with latitude and longitude and a text description. Because the POIs may not match the trajectory points, we map each POI to its nearest node in road network and regard the POI as an object in a semantic trajectory. In BTD, we use a real taxi trajectory data set collected by the T-drive project [42]. The original trajectories in BTD are very long since each of them contains all trips during a particular time period, which can be a couple of days. We divide these trajectories into half-an-hour sub-trajectories. The intent is to create trips with a realistic length and duration. To augment each trajectory point with text description, we randomly select a tweet from a collection of real-life tweets that comprises 2 million tweets and associate the text description of the tweet with the trajectory point.

2) Implementations: In the experiments, semantic trajectories and indexing structures are memory resident. All algorithms are implemented in Java and run on a cluster with 10 data nodes. Each node is equipped with two Intel ${ }^{\circledR}$ Xeon ${ }^{\circledR}$ Processors E5-2620 v3 (2.4GHz) and 128GB RAM. We use $\mathrm{CPU}$ time and the number of visited trajectories are our metrics of performance evaluation. In multi-threaded execution, the total runtime is the maximum runtime among all individual threads.

We study the performance of the non-self joins, i.e., $T \neq$ $Q$, and self joins, i.e., $T=Q$, in Sections $\mathrm{V}-\mathrm{B}$ and $\mathrm{V}-\mathrm{C}$, respectively. Semantic trajectories in $T$ and $Q$ are selected randomly from the datasets.

We evaluate the following three methods:

- $\quad$ STIF: Search with trajectory inverted file (Section III);

- $\quad \underline{\text { STM: }}$ Semantic trajectory summarization with trajectory pair pruning (Sections IV-B and IV-C);

- BFJoin: BFJoin algorithm with trajectory grouping and batch filtering (Sections IV-D).

When evaluating the number of trajectory visits, we do not report the performance of STM because STM and STIF incur the same numbers of trajectory visits. The parameter settings are listed in Table I.

\section{B. Experiment Results of Non-self Join}

1) Effect of the number of trajectories: Figures 8 and 9 show the effect of semantic trajectory cardinalities $|T|$ and $|Q|$, respectively, on the performance of the three algorithms. Intuitively, a larger $|T|$ (or $|Q|$ ) causes more trajectory pairs to be evaluated, which denotes that both CPU time and the number of visited trajectories are expected to be higher for

\footnotetext{
${ }^{12} \mathrm{https} / / /$ publish.illinois.edu/dbwork/open-data/

${ }^{13} \mathrm{https} / / /$ catalog.data.gov/dataset/points-of-interest-4aea0
} 


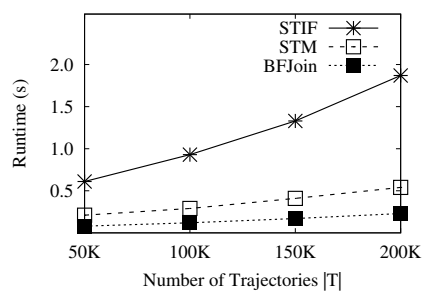

(a) BTD

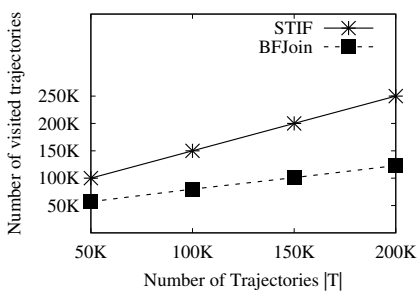

(b) BTD

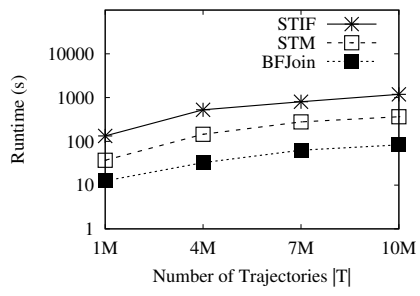

(c) NTD

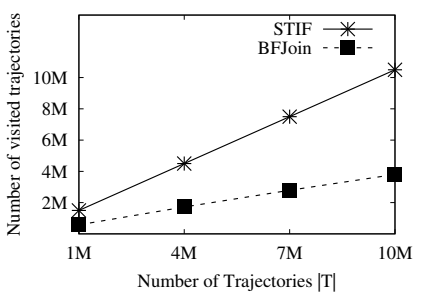

(d) NTD

Fig. 8. Effect of the number of trajectories $|T|$

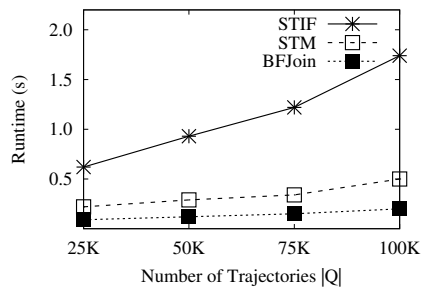

(a) BTD

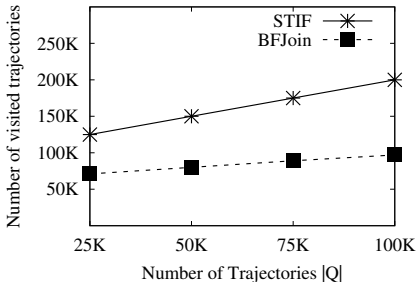

(b) BTD

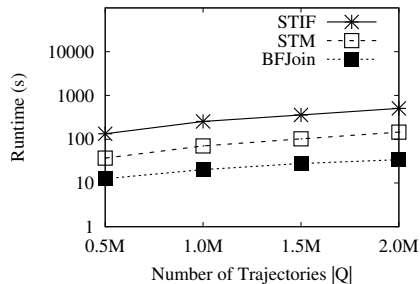

(c) NTD

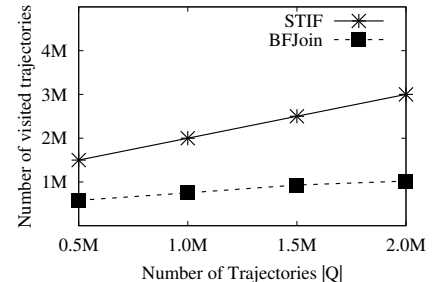

(d) NTD

Fig. 9. Effect of the number of trajectories $|Q|$

TABle I: PARAmeter Settings

\begin{tabular}{|c|c|c|}
\hline & NTD & BTD \\
\hline $\begin{array}{l}\text { Cardinality of tra- } \\
\text { jectories }|T|\end{array}$ & $\begin{array}{l}1,000,000- \\
10,000,000 / \text { default } \\
1,000,000\end{array}$ & $\begin{array}{l}50,000-200,000 \\
\text { default } 100,000\end{array}$ \\
\hline $\begin{array}{l}\text { Cardinality of tra- } \\
\text { jectories }|Q|\end{array}$ & $\begin{array}{l}500,000-2,000,000 / \\
\text { default } 500,000\end{array}$ & $\begin{array}{l}25,000-100,000 \\
\text { default } 50,000\end{array}$ \\
\hline $\begin{array}{l}\text { Similarity thresh- } \\
\text { old } \theta\end{array}$ & $\begin{array}{l}1.8-1.95 / \text { default } \\
1.9\end{array}$ & $\begin{array}{l}1.8-1.95 \text { / default } \\
1.9\end{array}$ \\
\hline $\begin{array}{l}\text { Preference param- } \\
\text { eter } \alpha\end{array}$ & $0.3-0.9$ / default 0.5 & $\begin{array}{l}0.3-0.9 / \text { default } \\
0.5\end{array}$ \\
\hline $\begin{array}{l}\text { MBR diagonal } \\
\text { threshold } \theta_{\text {diag }}\end{array}$ & 5-20 / default 10 & 5-20 / default 10 \\
\hline $\begin{array}{l}\text { Side length of grid } \\
\text { cell }\end{array}$ & $0.5-2$ / default: 1 & $0.5-2$ / default: 1 \\
\hline Thread count & $24-120 /$ default 24 & $24-120 /$ default 24 \\
\hline
\end{tabular}

all algorithms. We see that STM performs substantially better than STIF, which underscores the efficacy of our trajectory pair pruning scheme (cf. Sections IV-B and IV-C). In addition, our BFJoin algorithm further improves the CPU time by a factor of 1.8-2.2 (cf. Figures 8(a), 8(c), 9(a), and 9(c)), showing that a substantial proportion of trajectory pairs are filtered out during the intra-list batch filtering and trajectory-batch filtering phases. From Figure 8(c), we see that the BFJoin is able to process 1 million semantic trajectories $(|T|=1 \mathrm{M}$ and $|Q|=$ $0.5 \mathrm{M})$ in 12.5 seconds and 10 million semantic trajectories $(|T|=10 \mathrm{M}$ and $|Q|=0.5 \mathrm{M})$ in 85 seconds on default 24 threads. In contrast, the STIF algorithm takes 133 and 1,190 seconds in processing 1 million and 10 million trajectories, respectively.

2) Effect of similarity threshold $\theta$ : This set of experiments investigates the effect of similarity threshold $\theta$. Figure 10 shows the results when we vary $\theta$. We see that STM and BFJoin perform better when we increase the value of $\theta$. In contrast, the performance of STIF remains constant as we vary $\theta$. The reasons can be explained as follow. For STM and BFJoin, a higher value of $\theta$ means that more trajectory pairs may be pruned by our trajectory pair pruning scheme. As a consequence, both CPU time and the number of visited trajectories may decrease. In particular, we find that BFJoin is more sensitive to $\theta$ in comparison to STM (Figure 10(c)). This can be explained by the fact that a higher value of $\theta$ may increase the pruning power of both intra-list batch filtering and trajectory-batch filtering as more trajectory batches may be filtered out when we increase $\theta$.

3) Effect of preference parameter $\alpha$ : Figure 11 shows the effect of varying the preference parameter $\alpha$, which balances the importance of spatial similarity versus text similarity. The importance of the spatial similarity increases as we vary $\alpha$ from 0.3 to 1.0 . In particular, when $\alpha=1.0$, the similarity between two trajectories is solely measured by the spatial proximity. We see that the value of $\alpha$ has little influence on the performances of STIF and STM. STIF has no pruning scheme, and we need to compute the exact similarity of each pair of semantic trajectories in an inverted list. Thus, its performance is independent of $\alpha$. Next STM has a trajectory pair pruning scheme, which considers both the spatial and textual aspects. The observed performance of STM suggests that our trajectory pair pruning scheme is robust to imbalanced weights between spatial and text similarity. In contrast, BFJoin is moderately sensitive to $\alpha$. The reason is that the intra-batch filtering technique only considers the spatial aspect. Putting less weight on spatial similarity (i.e., decreasing $\alpha$ ) may lower the power of intra-batch filtering. Nevertheless, our trajectorybatch filtering technique, followed with intra-batch filtering, takes both spatial and textual aspects into account. As a result, the performance of BFJoin is still moderately better than STM even if we set $\alpha$ to 0.3 .

4) Effect of the side length of grid cell: We proceed to evaluate the effect of varying the grid granularity in trajectory 


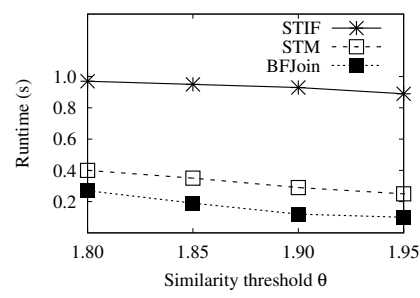

(a) BTD

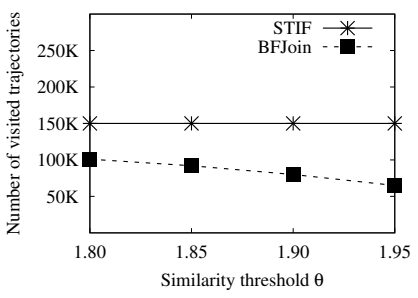

(b) BTD

Fig. 10. Effect of spatio-textual similarity threshold $\theta$

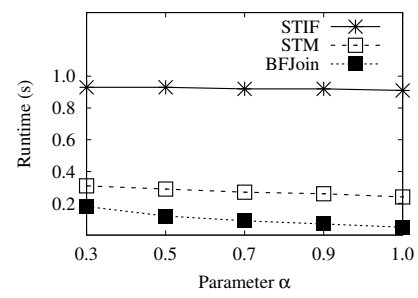

(a) BTD

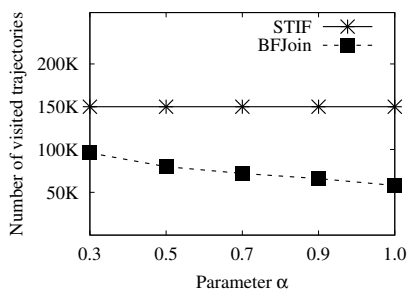

(b) BTD

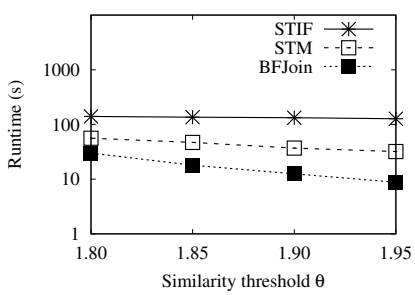

(c) NTD

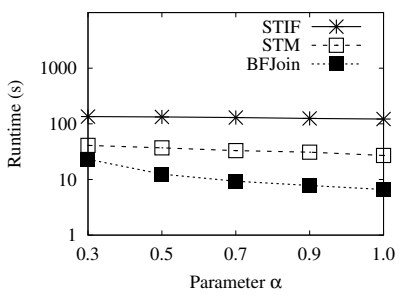

(c) NTD

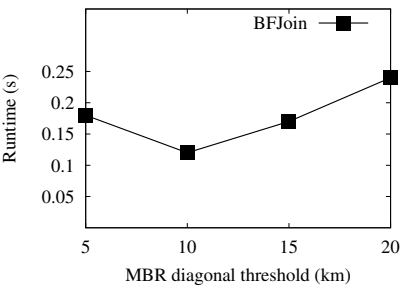

(a) BTD

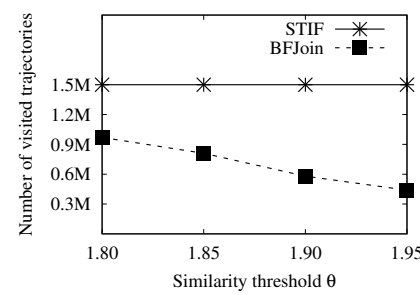

(d) NTD

Fig. 11. Effect of preference parameter $\alpha$

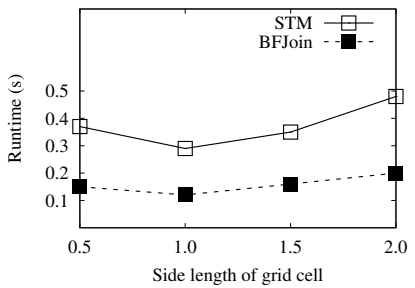

(a) BTD

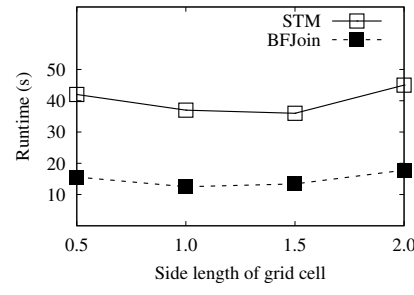

(b) NTD

Fig. 12. Effect of the side length of grid cell

pair pruning technique. From Figure 12, we find that both STM and BFJoin exhibit the best performance when the side length of grid cell is set between 1 to 1.5. A finer granularity can improve the accuracy of the spatial similarity upper bound between two trajectories (i.e., $\mathrm{ST}_{S}\left(\tau_{i}, \tau_{j}\right) . u b$, see Section IV-C). However, the time cost of computing the upper bound may increase accordingly. On the contrary, a coarser granularity may improve the efficiency of upper bound computation at the cost of lowering the accuracy.

\section{5) Effect of MBR diagonal length threshold $\theta_{\text {diag }}$ :}

TABLE IV: AVERAGE MBR SIZE IN PROPORTION TO THE TOTAL MAP SIZE

\begin{tabular}{|c|c|c|c|c|}
\hline $\begin{array}{r}\text { MBR diagonal } \\
\text { length (km) }\end{array}$ & 5 & 10 & 15 & 20 \\
\hline Bataset & $0.46 \%$ & $1.5 \%$ & $3.8 \%$ & $6.1 \%$ \\
\hline NTD & $0.22 \%$ & $0.83 \%$ & $1.7 \%$ & $2.8 \%$ \\
\hline
\end{tabular}

Figure 13 shows the effect of varying the MBR diagonal length threshold, $\theta_{\text {diag }}$, which is used in Algorithm 6. Table IV reports the average MBR size in proportion to the MBR size of the entire road network when we vary $\theta_{\text {diag }}$ from $5 \mathrm{~km}$ to $20 \mathrm{~km}$. When $\theta_{\text {diag }}$ is low, we may have a large

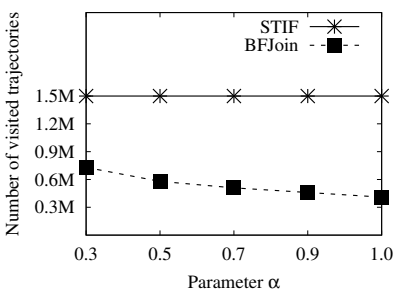

(d) NTD

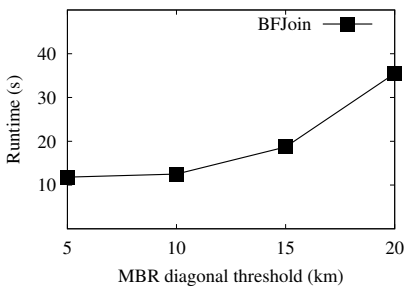

(b) NTD

Fig. 13. Effect of MBR diagonal length threshold $\theta_{\text {diag }}$

number of "small" batches associated with each inverted list. If we increase $\theta_{\text {diag }}$, we will have fewer trajectory batches. However, the average MBR diagonal length of each group may increase, which lowers the accuracy of the similarity upper bound between two batches (Equation 6) and the similarity upper bound between a trajectory and a batch (Equation 7).

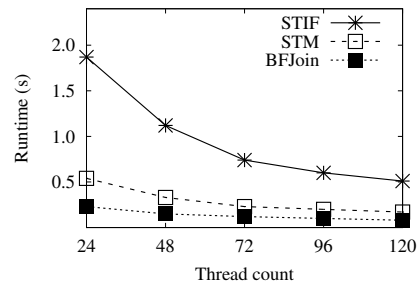

(a) BTD

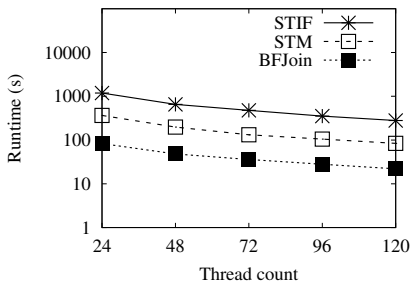

(b) NTD

Fig. 14. Effect of thread counts

6) Effect of thread counts: We study the effect of thread count on the efficiency of the algorithms using large trajectory data sets $(|T|=200 \mathrm{~K}$ and $|Q|=50 \mathrm{~K}$ for BTD, and $|T|$ $=10 \mathrm{M}$ and $|Q|=500 \mathrm{~K}$ for NTD). Figure 14 show that BFJoin consistently outperforms STIF by a factor of 8-12 and 
outperforms STM by $50 \%-75 \%$ regarding CPU time. In BTD, when we set the thread counts to 120 , BFJoin is able to solve the STS-Join problem over a collection of $200 \mathrm{~K}$ trajectories in 0.1 second, while in NTD, BFJoin is able to solve the STSJoin problem with $10 \mathrm{M}$ trajectories in 25 seconds.

\section{Experiment Results of Self Join}

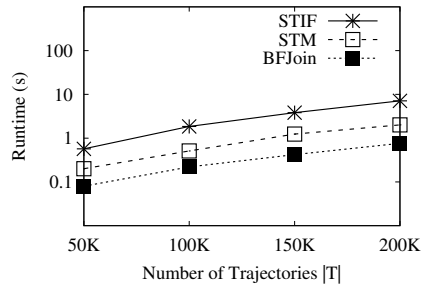

(a) BTD

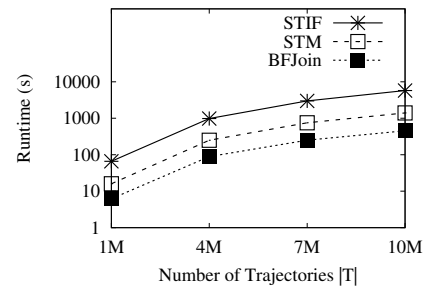

(b) NTD
Fig. 15. Effect of the number of semantic trajectories (self-join)

Figure 15 shows the runtime for the self joins when varying the trajectory cardinality. Note that the thread counts are set to 24 and 120 on BTD and NTD, respectively. The trends are similar to those of the non-self join. The BFJoin outperforms STIF by a factor of $\sim 8$ and by a factor of $\sim 11$ on BTD and NTD, respectively.

\section{RELATED WORK}

Spatial keyword trajectory query processing: A spatial keyword trajectory query contains a set of query locations and a set of keywords. Given a collection of semantic trajectories, the query finds a subset of trajectories that are spatio-textually similar to the query. The problem of efficient processing of top- $k$ spatial keyword trajectory query is studied by Cong et al. [13] and Zheng et al. [47]-[49] and Shang et al. [34]. Given a set $T$ of semantic trajectories and a query $q$ that includes a set of query locations and a set of keywords, a top- $k$ spatial keyword trajectory query ( $k \mathrm{SK}$ ) finds $k$ semantic trajectories from $T$ that have the shortest minimum match distances to $q$. In particular, the minimum match distance considers (1) the distance between query locations and a trajectory and (2) the number of POIs in the trajectory that cover the query keywords. Besides, Han et al. [17] focus on processing spatial keyword range trajectory query over semantic trajectory data. The query consists of query ranges and a set of keywords. It finds all trajectories that locate within the query ranges and collectively contain query keywords.

These proposals cannot process the STS-Join due to three reasons. (1) Different problems and matching schemes (query vs. join): The spatial keyword trajectory query contains a set of locations and a set of keywords, which means that the text information is not associated with the locations. In contrast, each object in a semantic trajectory contains both location text information, indicating that they are associated with each other. As a result, the query-trajectory matching scheme is totally different our trajectory-trajectory matching scheme. (2) Parallel processing: none of the above proposals are developed for parallel processing. Hence, they are incapable of handling very large semantic trajectory data sets.
Spatial keyword search and join: Given two collections of geo-textual objects $T$ and $Q$, the problem of spatial keyword join is to find all pairs of objects respectively from $R$ and $S$ that are both spatially close and textually similar. Liu et al. [16], [20] and $\mathrm{Hu}$ et al. [19] develop a filter-and-refine framework by utilizing spatial and textual signatures. Bouros et al. [3] propose a batch processing mechanism that dynamically partitions the geo-textual objects into groups according to their spatial and textual information. Rao et al. [28] develop two spatial-first and two text-first indexing schemes for efficient spatial-textual joint search. Either grid or Quad-tree is used for spatial indexing part. Zhang et al. [45] develop solutions for spatio-textual join under MapReduce framework by reducing the duplicates of data and the workload of the reducers. However, these proposals focus on object-object matching, which is different from our problem of trajectory-trajectory matching. Additionally, spatial keyword search is extensively investigated by existing studies [4], [7], [23], [24]. Given a collection of static or a stream of dynamic geo-textual objects, the problem is to find a subset of geo-textual objects (e.g., [25], [26], [41]) or summarized results (e.g., [8], [9], [37], [46]) based on a query with both spatial and textual requirements.

Traditional trajectory search and join: Trajectory join without text information is widely studied in literature [1], [2], [6], [11], [14], [29]-[33], [35], [36], [38]. The applications of trajectory similarity joins include trajectory near-duplicate detection, data cleaning, ridesharing recommendation, and traffic congestion prediction. Developing such joins typically involves a definition step and a query processing step. First, a similarity function is defined to evaluate the spatial and temporal similarities between two trajectories. Second, an efficient algorithm is developed to retrieve all trajectory pairs whose similarity satisfies a pre-defined threshold. However, these studies do not take text domain into consideration when calculating the similarity between two trajectories.

\section{CONCLUSIONS}

We studied a novel semantic trajectory similarity join (STSJoin), which targets a number of useful applications such as term-based trajectory duplicate detection, geo-text data cleaning, personalized ridesharing/carpooling recommendation, and itinerary recommendation. To process the STS-Join efficiently, we developed a number of trajectory pair filtering techniques that took into account the parallel processing capabilities of modern processors. We also proposed an efficient divide-andconquer algorithm for computing an upper bound of spatiotextual similarity between two semantic trajectories. The performance of the STS-Join was investigated through extensive experiments on large trajectory data. Experimental result has shown that the pruning power of our filtering techniques were strong enough and our join algorithm was capable of achieving high efficiency and scalability on massive-scale trajectory data.

\section{ACKNOWLEDGEMENTS}

This work was supported by the National Natural Science Foundation of China (61932004, 61922054, 61872235, 61729202, 61832017, U1636210) and the National Key Research and Development Program of China (2018YFC1504504, 2016YFB0700502). 


\section{REFERENCES}

[1] P. Bakalov, M. Hadjieleftheriou, E. J. Keogh, and V. J. Tsotras. Efficient trajectory joins using symbolic representations. In $M D M$, pages 86-93, 2005.

[2] P. Bakalov and V. J. Tsotras. Continuous spatiotemporal trajectory joins. In GSN, pages 109-128, 2006.

[3] P. Bouros, S. Ge, and N. Mamoulis. Spatio-textual similarity joins PVLDB, 6(1):1-12, 2012.

[4] X. Cao, L. Chen, G. Cong, C. S. Jensen, Q. Qu, A. Skovsgaard, D. Wu, and M. L. Yiu. Spatial keyword querying. In ER, volume 7532, pages 16-29. Springer, 2012

[5] L. Chen, G. Cong, C. S. Jensen, and D. Wu. Spatial keyword query processing: An experimental evaluation. PVLDB, 6(3):217-228, 2013.

[6] L. Chen, S. Shang, C. S. Jensen, B. Yao, Z. Zhang, and L. Shao. Effective and efficient reuse of past travel behavior for route recommendation. In $K D D$, pages $488-498$.

[7] L. Chen, S. Shang, C. Yang, and J. Li. Spatial keyword search: a survey. GeoInformatica, 24(1):85-106, 2020.

[8] L. Chen, S. Shang, Z. Zhang, X. Cao, C. S. Jensen, and P. Kalnis Location-aware top-k term publish/subscribe. In ICDE, pages 749-760.

[9] L. Chen, S. Shang, K. Zheng, and P. Kalnis. Cluster-based subscription matching for geo-textual data streams. In ICDE, pages 890-901.

[10] L. Chen, J. Xu, X. Lin, C. S. Jensen, and H. Hu. Answering why-not spatial keyword top-k queries via keyword adaption. In ICDE, pages 697-708, 2016.

[11] Y. Chen and J. M. Patel. Design and evaluation of trajectory join algorithms. In ACM-GIS, pages 266-275, 2009.

[12] Z. Chen, H. T. Shen, X. Zhou, Y. Zheng, and X. Xie. Searching trajectories by locations: an efficiency study. In SIGMOD, pages 255266, 2010.

[13] G. Cong, H. Lu, B. C. Ooi, D. Zhang, and M. Zhang. Efficient spatial keyword search in trajectory databases. arXiv:1205.2880, pages 1-12, 2012.

[14] H. Ding, G. Trajcevski, and P. Scheuermann. Efficient similarity join of large sets of moving object trajectories. In TIME, pages 79-87, 2008.

[15] C. Doulkeridis, A. Vlachou, D. Mpestas, and N. Mamoulis. Parallel and distributed processing of spatial preference queries using keywords. In EDBT, pages 318-329, 2017.

[16] J. Fan, G. Li, L. Zhou, S. Chen, and J. Hu. SEAL: spatio-textual similarity search. PVLDB, 5(9):824-835, 2012.

[17] Y. Han, L. Wang, Y. Zhang, W. Zhang, and X. Lin. Spatial keyword range search on trajectories. In DASFAA, pages 223-240, 2015.

[18] H. Hu, Y. Liu, G. Li, J. Feng, and K. Tan. A location-aware publish/subscribe framework for parameterized spatio-textual subscriptions. In $I C D E$, pages 711-722, 2015.

[19] H. Huiqi, L. Guoliang, B. Zhifeng, F. Jianhua, W. Yongwei, G. Zhiguo, and $\mathrm{X}$. Yaoqing. Top-k spatio-textual similarity join. IEEE Trans. Knowl. Data Eng., 28(2):551-565, 2016.

[20] S. Liu, G. Li, and J. Feng. Star-join: spatio-textual similarity join. In CIKM, pages 2194-2198, 2012.

[21] S. Liu, G. Li, and J. Feng. A prefix-filter based method for spatiotextual similarity join. IEEE Trans. Knowl. Data Eng., 26(10):23542367,2014

[22] Y. Lu, J. Lu, G. Cong, W. Wu, and C. Shahabi. Efficient algorithms and cost models for reverse spatial-keyword $k$-nearest neighbor search ACM Trans. Database Syst., 39(2):13:1-13:46, 2014.

[23] A. Magdy, L. Abdelhafeez, Y. Kang, E. Ong, and M. F. Mokbel. Microblogs data management: a survey. VLDB J., 29(1):177-216, 2020

[24] A. R. Mahmood and W. G. Aref. Scalable Processing of SpatialKeyword Queries. Synthesis Lectures on Data Management. Morgan \& Claypool Publishers, 2019.

[25] A. R. Mahmood, W. G. Aref, A. M. Aly, and M. Tang. Atlas: on the expression of spatial-keyword group queries using extended relational constructs. In SIGSPATIAL, pages 45:1-45:10.

[26] K. Mouratidis, J. Li, Y. Tang, and N. Mamoulis. Joint search by social and spatial proximity. IEEE Trans. Knowl. Data Eng., 27(3):781-793, 2015 .
[27] C. Parent, S. Spaccapietra, C. Renso, G. L. Andrienko, N. V. Andrienko, V. Bogorny, M. L. Damiani, A. Gkoulalas-Divanis, J. A. F. de Macêdo, N. Pelekis, Y. Theodoridis, and Z. Yan. Semantic trajectories modeling and analysis. ACM Comput. Surv., 45(4):42:1-42:32, 2013.

[28] J. Rao, J. Lin, and H. Samet. Partitioning strategies for spatio-textual similarity join. In BigSpatial@SIGSPATIAL, pages 40-49, 2014.

[29] S. Ray, A. D. Brown, N. Koudas, R. Blanco, and A. K. Goel. Parallel inmemory trajectory-based spatiotemporal topological join. In Big Data, pages 361-370. IEEE, 2015.

[30] S. Shang, L. Chen, C. S. Jensen, J. Wen, and P. Kalnis. Searching trajectories by regions of interest. IEEE Trans. Knowl. Data Eng., 29(7):1549-1562, 2017

[31] S. Shang, L. Chen, Z. Wei, C. S. Jensen, K. Zheng, and P. Kalnis. Trajectory similarity join in spatial networks. PVLDB, 10(11):11781189, 2017.

[32] S. Shang, L. Chen, Z. Wei, C. S. Jensen, K. Zheng, and P. Kalnis. Parallel trajectory similarity joins in spatial networks. VLDB J., 27(3):395-420, 2018.

[33] S. Shang, L. Chen, K. Zheng, C. S. Jensen, Z. Wei, and P. Kalnis Parallel trajectory-to-location join. IEEE Trans. Knowl. Data Eng., 31(6):1194-1207, 2019.

[34] S. Shang, R. Ding, B. Yuan, K. Xie, K. Zheng, and P. Kalnis. User oriented trajectory search for trip recommendation. In $E D B T$, pages 156-167, 2012.

[35] S. Shang, R. Ding, K. Zheng, C. S. Jensen, P. Kalnis, and X. Zhou. Personalized trajectory matching in spatial networks. VLDB J., 23(3):449468, 2014.

[36] Z. Shang, G. Li, and Z. Bao. DITA: distributed in-memory trajectory analytics. In SIGMOD, pages 725-740, 2018.

[37] A. Skovsgaard, D. Sidlauskas, and C. S. Jensen. Scalable top-k spatiotemporal term querying. In ICDE, pages 148-159.

[38] N. Ta, G. Li, Y. Xie, C. Li, S. Hao, and J. Feng. Signature-based trajectory similarity join. IEEE Trans. Knowl. Data Eng., 29(4):870883, 2017.

[39] P. Tampakis, C. Doulkeridis, N. Pelekis, and Y. Theodoridis. Distributed subtrajectory join on massive datasets. CoRR, abs/1903.07748, 2019.

[40] X. Wang, W. Zhang, Y. Zhang, X. Lin, and Z. Huang. Top-k spatialkeyword publish/subscribe over sliding window. VLDB J., 26(3):301326, 2017.

[41] C. Yang, L. Chen, S. Shang, F. Zhu, L. Liu, and L. Shao. Toward efficient navigation of massive-scale geo-textual streams. In IJCAI, pages 4838-4845.

[42] J. Yuan, Y. Zheng, X. Xie, and G. Sun. T-drive: Enhancing driving directions with taxi drivers' intelligence. IEEE Trans. Knowl. Data Eng., 25(1):220-232, 2013.

[43] C. Zhang, Y. Zhang, W. Zhang, and X. Lin. Inverted linear quadtree: Efficient top k spatial keyword search. In ICDE, pages 901-912, 2013.

[44] D. Zhang, K. Tan, and A. K. H. Tung. Scalable top-k spatial keyword search. In EDBT, pages 359-370, 2013.

[45] Y. Zhang, Y. Ma, and X. Meng. Efficient spatio-textual similarity join using mapreduce. In WI-IAT, pages 52-59, 2014.

[46] K. Zhao, L. Chen, and G. Cong. Topic exploration in spatio-temporal document collections. In SIGMOD, pages 985-998, 2016.

[47] B. Zheng, N. J. Yuan, K. Zheng, X. Xie, S. W. Sadiq, and X. Zhou. Approximate keyword search in semantic trajectory database. In $I C D E$, pages 975-986, 2015

[48] K. Zheng, S. Shang, N. J. Yuan, and Y. Yang. Towards efficient search for activity trajectories. In ICDE, pages 230-241, 2013.

[49] K. Zheng, B. Zheng, J. Xu, G. Liu, A. Liu, and Z. Li. Popularityaware spatial keyword search on activity trajectories. World Wide Web, 20(4):749-773, 2017. 